\title{
Robust Fault Detection of Linear Uncertain Time-Delay Systems Using Unknown Input Observers
}

\author{
Saeed Ahmadizadeh, ${ }^{1}$ Jafar Zarei, ${ }^{2}$ and Hamid Reza Karimi ${ }^{3}$ \\ ${ }^{1}$ Electrical Engineering Department, Iran University of Science and Technology, Narmak, Tehran 16846, Iran \\ ${ }^{2}$ Department of Electrical and Electronic Engineering, Shiraz University of Technology, Shiraz 71557-13876, Iran \\ ${ }^{3}$ Department of Engineering, Faculty of Engineering and Science, University of Agder, 4898 Grimstad, Norway
}

Correspondence should be addressed to Hamid Reza Karimi; hamid.r.karimi@uia.no

Received 18 July 2013; Accepted 13 September 2013

Academic Editor: Baocang Ding

Copyright (C) 2013 Saeed Ahmadizadeh et al. This is an open access article distributed under the Creative Commons Attribution License, which permits unrestricted use, distribution, and reproduction in any medium, provided the original work is properly cited.

This paper deals with the problem of fault detection for linear uncertain time-delay systems. The proposed method for Luenberger observers is developed for unknown input observers (UIOs), and a novel procedure for the design of residual based on UIOs is presented. The design procedure is carried out based on the $H_{\infty}$ model matching approach which minimizes the difference between generated residuals by the optimal observer and those by the designed observer in the presence of uncertainties. The optimal observer is designed for the ideal system and works so that the fault effect is maximized while the exogenous disturbances and noise effects are minimized. This observer can give disturbance decoupling in the presence of noise and uncertainties for linear uncertain time-delay systems. The developed method is applied to a numerical example, and the simulation results show that the proposed approach is able to detect faults reliably in the presence of modeling errors, disturbances, and noise.

\section{Introduction}

Fault detection and isolation (FDI) is an essential and challenging problem in many industrial applications. Among the various reported methods, much attention has been paid to model based approaches in the field of control engineering in recent years. For example, fault detection problem for discrete-time Markov jump systems and switched systems is investigated in $[1,2]$, respectively. The problem of fault reconstruction for a class of descriptor linear systems using sliding mode observers is presented in [3]. The sliding mode observers have been designed such that the actuator fault can be reconstructed using output measurements. The datadriven scheme for FDI is presented in [4] which exploits an adaptive residual generator and a bank of isolation observers. The designed scheme obtains observer parameters without identification of complete process model.
However, model based approaches are based on some idealized assumptions, one of which is that the mathematical model of the plant is a faithful replica of the plant dynamics [5]. As the mathematical model of a plant hardly represents its complete behavior, due to the existence of model uncertainty, noise, and unknown disturbances, it is essential to design a fault diagnosis system to take these effects into consideration. Motivated by the abovementioned issues, a robust fault detection scheme is exploited to design fault detection systems so that high sensitivity to faults as well as low sensitivity to uncertainties and perturbation can be obtained. Optimization techniques are widely used to solve this problem. One of the commonly used approaches to design such FDI scheme is representing the design procedure by $H_{\infty}$ and $H_{-}$ performance indexes. The main advantage of this approach is that it can be solved by linear matrix inequality (LMI) [6]. In $[7,8]$, a two-step FDI design methodology is presented. 
In this methodology, the optimal fault detection filter (FDF) has firstly been designed, neglecting the existence of model uncertainty. Next, the FDF, which is used as residual generator, has been obtained via $H_{\infty}$ model matching technique. The same approach is used in [9]; however, different dynamics is considered for the FDF.

Time delay is an inherent characteristic of many industrial systems; therefore, robust FDI problem for LTI systems with time delay received great attention over recent decades, and numerous articles have been presented. One approach is to solve the formulated design procedure using the eigenstructure assignment approach in which the residual signal is thoroughly decoupled from delay-free unknown input. Then the effect of the unknown input is minimized using $H_{\infty}$ norm [10]. In the presence of uncertainty, the same approach as delay-free case can be employed to obtain a robust FDI system. Indeed, solving the $H_{\infty}$ model matching problem results in achieving an FDF which acts the same as the optimal one [11-13]. Although the same approach is considered in these references, solving procedures are completely different owing to the difference between the dynamic of the filter and system. In [14], the problem of robust FDF design for the class of linear systems has been investigated. The system is subjected to mixed neutral and discrete time-varying delays and some nonlinear perturbations. The Luenberger type observer has been utilized to design FDF such that the residual signals effectively show fault occurrence.

Another approach commonly used to robust FDI scheme is to employ the unknown input observers (UIO), in which the residual is designed to be sensitive to faults but insensitive to unknown disturbances. Although the UIO has been widely used in estimation problems in both time delay and delayfree systems [15-18], there are few references that handle the problem of designing robust FDI $[19,20]$. In [20], a design procedure has been proposed for delay-free systems so that perturbations and exogenous signals have less effect on the residual signal and the fault has a detectable effect on the residual; however, the problem has not been presented in $H_{\infty}$ model matching technique. Motivated by this consideration, a robust FDF design using UIO for uncertain systems with time delay is presented and solved using $H_{\infty}$ model matching approach. In contrast to our previous work [18], we are concerned to design a robust FDF for the case in which the dynamic characteristic of fault signal is known. For this purpose, a two-step design procedure is developed. In the first step, the optimal fault detection based on UIO is designed for the system without uncertainty. Next, the UIO-based fault detection filter is approached to optimal one in the sense of $H_{\infty}$ norm. It is demonstrated through simulation that the presented fault detection observer is robust against uncertainty and sensitive enough to the faults.

Notation. The notations used throughout the paper are fairly standard. I and $\mathbf{0}$ represent identity matrix and zero matrix; the superscript " $T$ " stands for matrix transposition. $\|\cdot\|$ refers to the Euclidean vector norm or the induced matrix 2-norm. $\operatorname{diag}\{\cdot\}$ represents a block diagonal matrix.
The notation $P>0$ means that $P$ is real symmetric and positive definite; the symbol $*$ denotes the elements below the main diagonal of a symmetric block matrix.

\section{Problem Statement}

Many different industrial systems such as mechanical, electrical, meteorological, chemical, economic, and biological systems include time delay. In many studies linearized model of these systems around point of operation is considered. However, there are always some discrepancies between the real dynamics of the system and linearized model. These differences arise from systems uncertainty, as a consequence of neglecting dynamics, and changes in system parameters. Therefore, the following linear uncertain system with additive disturbances and time delay is considered to represent the described model:

$$
\begin{gathered}
\dot{x}(t)=(A+\Delta A(t)) x(t)+\left(A_{d}+\Delta A_{d}(t)\right) x(t-\tau) \\
+(B+\Delta B(t)) u(t)+E d(t)+F_{x} f(t)+R n(t), \\
y(t)=C x(t)+F_{y} f(t)+D n(t),
\end{gathered}
$$

where $x(t) \in \mathfrak{R}^{n}$ is the state vector, $y(t) \in \mathfrak{R}^{p}$ is the output vector, $u(t) \in \Re^{q}$ is the input vector, $d(t) \in \mathfrak{R}^{m}$ is an unknown scalar function representing the disturbance that belongs to $L_{2}^{m}(0, \infty), f(t) \in \mathfrak{R}^{f}$ denotes the faults, and $n(t) \in \mathfrak{R}^{r}$ represents the noise. Note that $\Delta A(t), \Delta B(t)$, and $\Delta A_{d}(t)$ are the norm bounded time-varying uncertainties of the matrices $A, B$, and $A_{d}$, respectively, and $\tau \geq 0$ is a constant delay. It is assumed that the characteristics of uncertainty matrices belong to

$$
\begin{gathered}
\Omega_{1}=\left\{\Delta A(t) \mid \Delta A(t)=M_{1} \Sigma_{1}(t) N_{1}, \Sigma_{1}^{T}(t) \Sigma_{1}(t) \leq I\right\}, \\
\Omega_{2}=\left\{\Delta B(t) \mid \Delta B(t)=M_{2} \Sigma_{2}(t) N_{2}, \Sigma_{2}^{T}(t) \Sigma_{2}(t) \leq I\right\}, \\
\Omega_{3}=\left\{\Delta A_{d}(t) \mid \Delta A_{d}(t)=M_{3} \Sigma_{3}(t) N_{3}, \Sigma_{3}^{T}(t) \Sigma_{3}(t) \leq I\right\},
\end{gathered}
$$

where $M_{i}$ and $N_{i}$ are predefined matrices. It is supposed that all over the paper the dimensions of matrices are compatible if they are not explicitly mentioned.

The dynamic characteristic of fault signal can be described by [21]

$$
\begin{gathered}
\dot{\theta}(t)=A_{\theta} \theta(t), \quad t \geq t_{f}, \\
\theta(t)=0, \quad t \in\left[0, t_{f}\right], \\
\theta\left(t_{f}\right)=\theta_{0}, \\
f(t)=F_{\theta} \theta(t),
\end{gathered}
$$

where $t_{f}$ is the time when a fault occurs and $A_{\theta}$ and $F_{\theta}$ are known matrices with appropriate dimensions. The initial time, $t_{f}$, and initial state, $\theta_{0}$, are supposed to be unknown. The dynamic equation (3) represents any fault with known 
dynamic characteristic and unknown amplitude and phase [21].

Unknown input observer for the class of time-delay system (1) is considered as [22]

$$
\begin{gathered}
\dot{z}(t)=F z+G z(t-\tau)+H u(t)+K_{1 z} y(t)+K_{2 z} y(t-\tau), \\
\widehat{x}(t)=z(t)+L_{1} y(t),
\end{gathered}
$$

where $\widehat{x}(t)$ is the estimated state vector. The dynamic of $\widehat{x}(t)$ is governed by

$$
\begin{aligned}
\dot{\hat{x}}(t)= & F \hat{x}+G \hat{x}(t-\tau)+H u(t)+L_{1} \dot{y}(t) \\
& +L_{2} y(t)+L_{3} y(t-\tau),
\end{aligned}
$$

where $F, G, H$, and $L_{1}$ are the observer matrices and $L_{2}=$ $K_{1 z}-F L_{1}, L_{3}=K_{2 z}-G L_{1}$.

The observer matrices will be designed such that the disturbance and input are decoupled from the estimation error defined by $e(t)=x(t)-\widehat{x}(t)$. When UIO-based filter defined by (4) is applied to the system described in (1), the state estimation error will be

$$
\begin{aligned}
\dot{e}(t)= & F e(t)+G e(t-\tau)+\left(\left(I-L_{1} C\right) A-L_{2} C-F\right) x(t) \\
& +\left(\left(I-L_{1} C\right) A_{d}-L_{3} C-G\right) x(t-\tau) \\
& +\left(\left(I-L_{1} C\right) B-H\right) u(t) \\
& +\left(I-L_{1} C\right) E d(t) \\
& +\left(\left(I-L_{1} C\right) F_{x} F_{\theta}-L_{2} F_{y} F_{\theta}-L_{1} F_{y} F_{\theta} A_{\theta}\right) \theta(t) \\
& -L_{3} F_{y} F_{\theta} \theta(t-\tau)+\left(\left(R-L_{1} C R\right)-L_{2} D\right) n(t) \\
& -L_{3} D n(t-\tau)-L_{1} D \dot{n}(t) \\
& +\left(I-L_{1} C\right) \Delta A(t) x(t)+\left(I-L_{1} C\right) \Delta A_{d}(t) x(t-\tau) \\
& +\left(I-L_{1} C\right) \Delta B(t) u(t) .
\end{aligned}
$$

In the absence of uncertainties and faults, it is shown that the observer, defined by (4), is UIO for the predefined system by (1) if the following conditions are satisfied [22].

Condition 1:

$\dot{e}(t)=F e(t)+G e(t-\tau)$ is asymptotically stable.

Condition 2:

$$
F=\left(I-L_{1} C\right) A-L_{2} C .
$$

Condition 3:

$$
G=\left(I-L_{1} C\right) A_{d}-L_{3} C
$$

Condition 4:

$$
H=\left(I-L_{1} C\right) B \text {. }
$$

Condition 5:

$$
\left(I-L_{1} C\right) E=\mathbf{0},
$$

where $\mathbf{0}$ denotes a null matrix with compatible dimension. Using these relationships, and considering definitions in (12), the state estimation error dynamic (6) is transformed to (13):

$$
\begin{gathered}
T=\left(I-L_{1} C\right) \\
\bar{F}=\left[\left(I-L_{1} C\right) F_{x} F_{\theta}-L_{2} F_{y} F_{\theta}-L_{1} F_{y} F_{\theta} A_{\theta}-L_{3} F_{y} F_{\theta}\right] \\
\bar{R}=\left[-L_{1} D\left(I-L_{1} C\right) R-L_{2} D-L_{3} D\right] \\
\bar{\theta}=\left[\theta^{T}(t) \theta^{T}(t-\tau)\right]^{T} \\
\bar{n}=\left[\dot{n}^{T}(t) n^{T}(t) n^{T}(t-\tau)\right]^{T} \\
\dot{e}(t)=F e(t)+G e(t-\tau)+\bar{F} \bar{\theta}+\bar{R} \bar{n} \\
+T \Delta A(t) x(t)+T \Delta A_{d}(t) x(t-\tau) \\
+T \Delta B(t) u(t) .
\end{gathered}
$$

In order to use the UIO for fault detection purposes, a residual signal should be defined. Difference between measured output and estimated output is usually considered as a residual signal. In current work, a more general form for residual reference signal is considered as follows:

$$
\begin{gathered}
\widehat{y}(t)=C \hat{x}(t), \\
r(t)=V_{1}(y(t)-\hat{y}(t))+V_{2}(y(t-\tau)-\hat{y}(t-\tau)) \\
=V_{1} C e(t)+V_{2} C e(t-\tau)+K_{1} \bar{\theta}(t)+K_{2} \bar{n}(t), \\
K_{1}=\left[\begin{array}{lll}
V_{1} F_{y} F_{\theta} & V_{2} F_{y} F_{\theta}
\end{array}\right], \\
K_{2}=\left[\begin{array}{lll}
\mathbf{0} & V_{1} D & V_{2} D
\end{array}\right] .
\end{gathered}
$$

The goal of robust fault detection problem is to minimize the performance index defined in (15) for all classes of model uncertainties belonging to $\Omega_{i}$. In general, this performance index is minimized using $H_{\infty}$ model matching approach which minimizes the difference between the residual signal $(r(t))$ and reference residual signal $\left(r_{f}(t)\right)$ 
in the presence of the worst case disturbance signals. This performance index has been minimized by the following steps.

Step 1. The ideal residual signal generator system has been designed for the system without uncertainty defined in (16). The residual signal shows the maximum sensitivity to the fault signal while it has the minimum sensitivity to disturbance, noise, and unknown inputs.

Step 2. The residual signal generator system has been designed such that the performance index (17) is minimized and the overall system (19) is asymptotically stable:

$$
\begin{gathered}
J_{r}=\min _{\left(\Delta A, \Delta B, \Delta A_{d}\right) \in \Omega_{i}} \frac{\left\|G_{r[d \bar{n}]}\right\|_{\infty}}{\left\|G_{r \bar{f}}\right\|_{\infty}} \\
\dot{e}_{f}(t)=F^{*} e_{f}(t)+G^{*} e_{f}(t-\tau)+\bar{F}^{*} \bar{\theta}(t)+\bar{R}^{*} \bar{n}(t), \\
r_{f}(t)=V_{1}^{*} C e_{f}(t)+V_{2}^{*} C e_{f}(t-\tau)+K_{1}^{*} \bar{\theta}(t)+K_{2}^{*} \bar{n}(t), \\
J_{r_{e}}=\sup _{\left(\Delta A, \Delta B, \Delta A_{d}\right) \in \Omega_{i}} \frac{\left\|r_{e}\right\|_{2}}{\|\omega\|_{2}}<\gamma,
\end{gathered}
$$

where

$$
\begin{aligned}
& r_{e}(t)=r(t)-r_{f}(t), \\
& \omega=\left[\begin{array}{llll}
u^{T} & \bar{\theta}^{T} & d^{T} & \bar{n}^{T}
\end{array}\right]^{T}, \\
& \dot{\zeta}(t)=(\widetilde{A}+\Delta \widetilde{A}) \zeta(t)+\left(\widetilde{A}_{d}+\Delta \widetilde{A}_{d}\right) \zeta(t-\tau) \\
& +\left(\widetilde{B}_{\omega_{1}}+\Delta \widetilde{B}_{\omega_{1}}\right) \omega(t) \\
& r_{e}(t)=\widetilde{C}_{1} \zeta(t)+\widetilde{C}_{2} \zeta(t-\tau)+\widetilde{D} \omega(t),
\end{aligned}
$$

where

$$
\begin{aligned}
& \zeta(t)=\left[\begin{array}{lll}
e^{T}(t) & e_{f}^{T}(t) & x^{T}(t)
\end{array}\right]^{T}, \\
& \widetilde{A}=\left[\begin{array}{ccc}
F & \mathbf{0} & \mathbf{0} \\
\mathbf{0} & F^{*} & \mathbf{0} \\
\mathbf{0} & \mathbf{0} & A
\end{array}\right], \quad \widetilde{A}_{d}=\left[\begin{array}{ccc}
G & \mathbf{0} & \mathbf{0} \\
\mathbf{0} & G^{*} & \mathbf{0} \\
\mathbf{0} & \mathbf{0} & A_{d}
\end{array}\right], \\
& \widetilde{B}_{\omega_{1}}=\left[\begin{array}{cccc}
\mathbf{0} & \bar{F} & \mathbf{0} & \bar{R} \\
\mathbf{0} & \bar{F}^{*} & \mathbf{0} & \bar{R}^{*} \\
B & K_{3} & E & K_{4}
\end{array}\right], \quad \Delta \widetilde{A}=\left[\begin{array}{ccc}
\mathbf{0} & \mathbf{0} & T \Delta A \\
\mathbf{0} & \mathbf{0} & \mathbf{0} \\
\mathbf{0} & \mathbf{0} & \Delta A
\end{array}\right],
\end{aligned}
$$

$$
\begin{aligned}
& \Delta \widetilde{A}_{d}=\left[\begin{array}{ccc}
\mathbf{0} & \mathbf{0} & T \Delta A_{d} \\
\mathbf{0} & \mathbf{0} & \mathbf{0} \\
\mathbf{0} & \mathbf{0} & \Delta A_{d}
\end{array}\right], \quad \Delta \widetilde{B}_{\omega_{1}}=\left[\begin{array}{cccc}
T \Delta B & \mathbf{0} & \mathbf{0} & \mathbf{0} \\
\mathbf{0} & \mathbf{0} & \mathbf{0} & \mathbf{0} \\
\Delta B & \mathbf{0} & \mathbf{0} & \mathbf{0}
\end{array}\right], \\
& \widetilde{C}_{1}=\left[\begin{array}{lll}
V_{1} C & -V_{1}^{*} C & 0
\end{array}\right], \quad \widetilde{C_{2}}=\left[\begin{array}{llll}
V_{2} C & -V_{2}^{*} C & 0
\end{array}\right], \\
& \widetilde{D}=\left[\begin{array}{lllll}
\mathbf{0} & K_{1}-K_{1}^{*} & \mathbf{0} & K_{2}-K_{2}^{*}
\end{array}\right], \\
& K_{3}=\left[\begin{array}{ll}
F_{x} F_{\theta} & \mathbf{0}
\end{array}\right], \quad K_{4}=\left[\begin{array}{lll}
\mathbf{0} & R & \mathbf{0}
\end{array}\right] .
\end{aligned}
$$

Furthermore, using (2) it is easy to see that $\Delta \widetilde{A}, \Delta \widetilde{A}_{d}, \Delta \widetilde{B}_{\omega_{1}}$ can be expressed by

$$
\begin{gathered}
\Delta \widetilde{A}=\widetilde{M}_{1} \Sigma_{1}(t) \widetilde{N}_{1}=\left[\begin{array}{c}
T M_{1} \\
\mathbf{0} \\
M_{1}
\end{array}\right] \Sigma_{1}(t)\left[\begin{array}{lll}
\mathbf{0} & \mathbf{0} & N_{1}
\end{array}\right], \\
\Delta \widetilde{A}_{d}=\widetilde{M}_{2} \Sigma_{2}(t) \widetilde{N}_{2}=\left[\begin{array}{c}
T M_{2} \\
\mathbf{0} \\
M_{2}
\end{array}\right] \Sigma_{2}(t)\left[\begin{array}{lll}
\mathbf{0} & \mathbf{0} & N_{2}
\end{array}\right], \\
\Delta \widetilde{B}_{\omega_{1}}=\widetilde{M}_{3} \Sigma_{3}(t) \widetilde{N}_{3}=\left[\begin{array}{c}
T M_{3} \\
\mathbf{0} \\
M_{3}
\end{array}\right] \Sigma_{3}(t)\left[\begin{array}{llll}
N_{3} & \mathbf{0} & \mathbf{0} & \mathbf{0}
\end{array}\right] .
\end{gathered}
$$

Before developing theorems that are utilized in designed procedure, the following lemmas, which are useful to prove the theorems, are introduced.

Lemma 1 (see [22]). Condition 5 is solvable if and only if the following relation holds:

$$
\operatorname{rank}(C E)=m, \quad m \leq p .
$$

The general solution of condition 5 can be calculated by

$$
\begin{aligned}
L_{1} & =E(C E)^{+}+Y\left[I-C E(C E)^{+}\right] \\
& =\Theta_{1}+Y \Theta_{2},
\end{aligned}
$$

where $Y$ is an arbitrary matrix with an appropriate dimension.

Lemma 2. Suppose that $M, N$, and $\Sigma(t)$ are compatible and $\Sigma^{T}(t) \Sigma(t) \leq I$; then there exists a scalar $\varepsilon>0$ such that the following equation holds:

$$
M \Sigma N+(M \Sigma N)^{T} \leq \varepsilon M M^{T}+\varepsilon^{-1} N^{T} N .
$$

(a) Reference Model Selection (Step 1). Reference model selection is an important key to the design of robust fault detection filter for linear uncertain time-delay systems. To this end, the analogues procedure as that for fault detection in [6] is extended for delay systems considering the UIO as the 
fault detection filter. According to (16), the reference residual signal can be written as sum of two signals, $r_{f \bar{n}}(t)$ and $r_{f \bar{\theta}}(t)$. The reference model should be chosen such that the effect of exogenous signals on the reference residual signal is minimized while the effect of fault signal is maximized. These two tasks are described mathematically by

$$
\begin{gathered}
\left\|T\left(r_{f \bar{n}}, \bar{n}\right)\right\|_{\infty} \leq \alpha, \\
\left\|T\left(r_{f \bar{f}}, \bar{f}\right)\right\|_{-} \geq \beta,
\end{gathered}
$$

where $T(\cdot, \cdot)$ is the transfer function between two signals. The following two theorems provide conditions which ensure the asymptotic stability of (16). They also provide the conditions that increase the sensitivity of the residual signal from faults and decrease the sensitivity of residual signal from noise.

Theorem 3. For given $\alpha>0$, if there exist symmetric positive matrices $P, Q, V_{1}^{*}, V_{2}^{*}, \Phi_{1}^{*}, \Phi_{2}^{*}$, and $\Phi_{3}^{*}$ such that the following LMI holds:

$$
\left[\begin{array}{cccc}
P F^{*}+F^{* T} P+Q & P G^{*} & P \bar{R}^{*} & C^{T} V_{1}^{* T} \\
* & -Q & \mathbf{0} & C^{T} V_{2}^{* T} \\
* & * & -\alpha^{2} I & K_{2}^{* T} \\
* & * & * & -I
\end{array}\right]<0,
$$

where

$$
\begin{gathered}
P F^{*}=P\left(A-\Theta_{1} C A\right)-\Phi_{1}^{*}\left(\Theta_{2} C A\right)-\Phi_{2}^{*} C, \\
P G^{*}=P\left(A_{d}-\Theta_{1} C A_{d}\right)-\Phi_{1}^{*}\left(\Theta_{2} C A_{d}\right)-\Phi_{3}^{*} C, \\
P \bar{R}^{*}=\left[-P\left(\Theta_{1} D\right)-\Phi_{1}^{*}\left(\Theta_{2} D\right) P\left(R-\Theta_{1} C R\right)-\Phi_{1}^{*}\left(\Psi_{2} C R\right)-\Phi_{2}^{*} D-\Phi_{3}^{*} D\right], \\
K_{2}^{*}=\left[\begin{array}{lll}
\mathbf{0} & V_{1}^{*} D & V_{2}^{*} D
\end{array}\right],
\end{gathered}
$$

then the system (28) is asymptotically stable and $\left\|T\left(r_{f \bar{n}}, \bar{n}\right)\right\|_{\infty} \leq \alpha$. Furthermore, the UIO matrices are obtained from conditions 2 to 5 , and $Y^{*}=P^{-1} \Phi_{1}, L_{2}=P^{-1} \Phi_{2}, L_{3}=$ $P^{-1} \Phi_{3}$,

$$
\begin{gathered}
\dot{e}_{f \bar{n}}(t)=F^{*} e_{f \bar{n}}(t)+G^{*} e_{f \bar{n}}(t-\tau)+\bar{R}^{*} \bar{n}(t), \\
r_{f \bar{n}}(t)=V_{1}^{*} C e_{f \bar{n}}(t)+V_{2}^{*} C e_{f \bar{n}}(t-\tau)+K_{2}^{*} \bar{n}(t) .
\end{gathered}
$$

Proof. Condition $\left\|T\left(r_{f \bar{n}}, \bar{n}\right)\right\|_{\infty} \leq \alpha$ is equivalent to $J_{r_{f \bar{n}}}$ : $\int_{0}^{\infty}\left(r_{f \bar{n}}^{T}(t) r_{f \bar{n}}(t)-\alpha^{2} \bar{n}^{T}(t) \bar{n}(t)\right) d t \geq 0$. Now Consider the
Lyapunov-Krasovskii function which is defined as $V(t)=$ $e_{f \bar{n}}^{T}(t) P e_{f \bar{n}}(t)+\int_{t-\tau}^{t} e_{f \bar{n}}^{T}(s) Q e_{f \bar{n}}(s) d s$. Then we have

$$
\begin{aligned}
J_{r_{f \bar{n}}} & \int_{0}^{\infty}\left(r_{f \bar{n}}^{T}(t) r_{f \bar{n}}(t)-\alpha^{2} \bar{n}^{T}(t) \bar{n}(t)+\dot{V}(t)\right) d t \\
& +V(0)-V(\infty) .
\end{aligned}
$$

Assume $r_{f \bar{n}}(t)=\mathbf{0}$ for $t \in[-\tau, 0]$. Since $V(\infty)>0$, it can be concluded that

$$
J_{r_{f \bar{n}}} \leq \int_{0}^{\infty}\left(r_{f \bar{n}}^{T}(t) r_{f \bar{n}}(t)-\alpha^{2} \bar{n}^{T}(t) \bar{n}(t)+\dot{V}(t)\right) d t .
$$

Taking derivative from $V(t)$ and considering (28) yield

$$
J_{r_{f \bar{n}}} \leq \int_{t-\tau}^{t}\left[\begin{array}{c}
e_{f \bar{n}}(t) \\
e_{f \bar{n}}(t-\tau) \\
\bar{n}(t)
\end{array}\right] \underbrace{\left[\begin{array}{ccc}
P F^{*}+F^{* T} P+Q+C^{T} V_{1}^{* T} V_{1}^{*} C & P G^{*}+C^{T} V_{1}^{* T} V_{2}^{*} C & P \bar{R}^{*}+C^{T} V_{1}^{* T} K_{2}^{*} \\
* & C^{T} V_{2}^{* T} V_{2}^{*} C-Q & C^{T} V_{2}^{* T} K_{2}^{*} \\
* & * & -\alpha^{2} I+K_{2}^{* T} K_{2}^{*}
\end{array}\right]}_{\Xi}\left[\begin{array}{c}
e_{f \bar{n}}(t) \\
e_{f \bar{n}}(t-\tau) \\
\bar{n}(t)
\end{array}\right] .
$$


Hence, $\Xi<0$ implies $J_{r_{f \bar{n}}}<0$. Using the Schur complement theorem (26) is concluded from (31). Indeed, the inequality (26), without considering (27), includes nonlinear terms of $P Y^{*}, P L_{2}^{*}$, and $P L_{3}^{*}$ which lead the LMI to be infeasible. To overcome this problem, define $\Phi_{1}^{*}=P Y^{*}$,
$\Phi_{2}^{*}=P L_{2}^{*}$, and $\Phi_{3}^{*}=P L_{3}^{*}$. Using conditions 2, 3 and (23) it can be shown that (27) makes the LMI feasible. It completes the proof.

Theorem 4. For given $\beta>0$, if there exists symmetric positive matrices $P, Q, V_{1}^{*}, V_{2}^{*}, \Phi_{1}^{*}$, $\Phi_{2}^{*}$, and $\Phi_{3}^{*}$ such that the following LMI holds:

$$
\left[\begin{array}{cccc}
P F^{*}+F^{* T} P-Q+2 \varphi_{1}\left(V_{1}^{*}, V_{1 c}^{n}\right) & -P G^{*} & -P \bar{F}^{*} & C^{T} V_{1}^{* T} \\
* & -Q+2 \varphi_{2}\left(V_{2}^{*}, V_{2 c}^{n}\right) & \mathbf{0} & C^{T} V_{2}^{* T} \\
* & * & \beta^{2} F_{\theta}^{T} F_{\theta}+2 \varphi_{3}\left(K_{1}^{*}, K_{1}^{n}\right) & K_{1}^{* T} \\
* & * & * & -I
\end{array}\right]<0,
$$

where

$$
\begin{gathered}
P F^{*}=P\left(A-\Theta_{1} C A\right)-\Phi_{1}^{*}\left(\Theta_{2} C A\right)-\Phi_{2}^{*} C, \\
P G^{*}=P\left(A_{d}-\Theta_{1} C A_{d}\right)-\Phi_{1}^{*}\left(\Theta_{2} C A_{d}\right)-\Phi_{3}^{*} C, \\
P \bar{F}^{*}=\left[P\left(F_{x} F_{\theta}-\Theta_{1} C F_{x} F_{\theta}\right)-\Phi_{1}^{*}\left(\Theta_{2} C F_{x} F_{\theta}\right)-\Phi_{2}^{*} F_{y} F_{\theta}-P\left(\Psi_{1} F_{y} F_{\theta} A_{\theta}\right)-\Phi_{1}^{*}\left(\Psi_{2} F_{y} F_{\theta} A_{\theta}\right)-\Phi_{3}^{*} F_{y} F_{\theta}\right], \\
K_{1}^{*}=\left[V_{1}^{*} F_{y} F_{\theta} V_{2}^{*} F_{y} F_{\theta}\right], \\
V_{1 c}^{n}=V_{1}^{* n-1} C, \quad V_{2 c}^{n}=V_{2}^{* n-1} C, \quad K_{11}^{n}=V_{1}^{* n-1} F_{y} F_{\theta}, K_{12}^{n}=V_{2}^{* n-1} F_{y} F_{\theta}, \quad \text { for } n=1,2, \ldots, \\
K_{1}^{n}=\left[\begin{array}{ll}
K_{11}^{n} & \left.K_{12}^{n}\right]
\end{array}\right] \\
\varphi_{1}\left(V_{1}^{*}, V_{1 c}^{n}\right)=\left(V_{1 c}^{n}\right)^{T} V_{1 c}^{n}-\left(V_{1 c}^{n}\right)^{T} V_{1}^{*} C-C^{T} V_{1}^{* T} V_{1 c}^{n}, \\
\varphi_{2}\left(V_{2}^{*}, V_{2 c}^{n}\right)=\left(V_{2 c}^{n}\right)^{T} V_{2 c}^{n}-\left(V_{2 c}^{n}\right)^{T} V_{2}^{*} C-C^{T} V_{2}^{* T} V_{2 c}^{n}, \\
\varphi_{3}\left(K_{1}^{*}, K_{1}^{n}\right)=\left(K_{1}^{n}\right)^{T} K_{1}^{n}-\left(K_{1}^{n}\right)^{T} V_{1}^{*} F_{y} F_{\theta}-F_{\theta}^{T} F_{y}^{T} V_{1}^{* T} K_{1}^{n},
\end{gathered}
$$

then the system (35) is asymptotically stable, and $\left\|T\left(r_{f \bar{\theta}}, \bar{f}\right)\right\|_{-} \geq \beta$. Moreover, the UIO matrices are obtained from conditions 2 to 5 , and $Y^{*}=P^{-1} \Phi_{1}, L_{2}=P^{-1} \Phi_{2}, L_{3}=$ $P^{-1} \Phi_{3}$,

$$
\begin{gathered}
\dot{e}_{f \bar{\theta}}(t)=F^{*} e_{f \bar{\theta}}(t)+G^{*} e_{f \bar{\theta}}(t-\tau)+\bar{F}^{*} \bar{\theta}(t), \\
r_{f \bar{\theta}}(t)=V_{1}^{*} C e_{f \bar{\theta}}(t)+V_{2}^{*} C e_{f \bar{\theta}}(t-\tau)+K_{2}^{*} \bar{\theta}(t) .
\end{gathered}
$$

Proof. Condition $\left\|T\left(r_{f \bar{f}}, \bar{f}\right)\right\|_{-} \geq \beta$ is equivalent to $J_{r_{f \bar{f}}}$ : $\int_{0}^{\infty}\left(r_{f \bar{\theta}}^{T}(t) r_{f \bar{\theta}}(t)-\beta^{2} \bar{f}^{T}(t) \bar{f}(t)\right) d t \geq 0$. Now consider the Lyapunov-Krasovskii function which is defined as $V(t)=$ $e_{f \bar{\theta}}^{T}(t) P e_{f \bar{\theta}}(t)+\int_{t-\tau}^{t} e_{f \bar{\theta}}^{T}(s) Q e_{f \bar{\theta}}(s) d s$. Then we have

$$
\begin{aligned}
J_{r_{f \bar{\theta}}}=\int_{0}^{\infty}\left(r_{f \bar{\theta}}^{T}(t) r_{f \bar{\theta}}(t)-\beta^{2} \bar{f}^{T}(t)\right. \\
\quad \times \bar{f}(t)-\dot{V}(t)) d t-V(0)+V(\infty) .
\end{aligned}
$$

Assume $r_{f \bar{\theta}}(t)=\mathbf{0}$ for $t \in[-\tau, 0]$. Since $V(\infty)>0$, we have

$$
J_{r_{f \bar{\theta}}} \geq \int_{0}^{\infty}\left(r_{f \bar{\theta}}^{T}(t) r_{f \bar{\theta}}(t)-\beta^{2} \bar{f}^{T}(t) \bar{f}(t)-\dot{V}(t)\right) d t .
$$

Taking derivative from $V(t)$ and considering (35) yield

$$
J_{r_{f \bar{\theta}}}>\int_{t-\tau}^{t}\left[\begin{array}{c}
e_{f \bar{\theta}}(t) \\
e_{f \bar{\theta}}(t-\tau) \\
\bar{\theta}(t)
\end{array}\right]^{T} \underbrace{\left[\begin{array}{ccc}
-P F^{*}-F^{* T} P-Q+C^{T} V_{1}^{* T} V_{1}^{*} C & -P G^{*}+C^{T} V_{1}^{* T} V_{2}^{*} C & -P \bar{F}^{*}+C^{T} V_{1}^{* T} K_{1}^{*} \\
* & C^{T} V_{2}^{* T} V_{2}^{*} C+Q & C^{T} V_{2}^{* T} K_{1}^{*} \\
* & * & -\beta^{2} F_{\theta}^{T} F_{\theta}+K_{1}^{* T} K_{1}^{*}
\end{array}\right]}_{\Xi}\left[\begin{array}{c}
e_{f \bar{\theta}}(t) \\
e_{f \bar{\theta}}(t-\tau) \\
\bar{\theta}(t)
\end{array}\right] .
$$


Hence, $\Xi>0$ implies $J_{r_{f \bar{\theta}}}>0 . \Xi>0$ is equivalent to

$$
\left[\begin{array}{ccc}
P F^{*}+F^{*^{T}} P+Q-C^{T} V_{1}^{* T} V_{1}^{*} C & -P G^{*}+C^{T} V_{1}^{* T} V_{2}^{*} C & -P \bar{F}^{*}+C^{T} V_{1}^{* T} K_{1}^{*} \\
* & -Q-C^{T} V_{2}^{* T} V_{2}^{*} C & C^{T} V_{2}^{* T} K_{1}^{*} \\
* & * & \beta^{2} F_{\theta}^{T} F_{\theta}-K_{1}^{* T} K_{1}^{*}
\end{array}\right]<0 .
$$

Then, (39) can be written as

$$
\left[\begin{array}{ccc}
P F^{*}+F^{* T} P+Q-2 C^{T} V_{1}^{* T} V_{1}^{*} C & -P G^{*}+C^{T} V_{1}^{* T} V_{2}^{*} C & -P \bar{F}^{*}+C^{T} V_{1}^{* T} K_{1}^{*} \\
* & -Q-2 C^{T} V_{2}^{* T} V_{2}^{*} C & C^{T} V_{2}^{* T} K_{1}^{*} \\
* & * & \beta^{2} F_{\theta}^{T} F_{\theta}-2 K_{1}^{* T} K_{1}^{*}
\end{array}\right]+\left[\begin{array}{lll}
C^{T} V_{1}^{* T} & C^{T} V_{2}^{* T} & K_{1}^{* T}
\end{array}\right] I\left[\begin{array}{c}
V_{1}^{*} C \\
V_{2}^{*} C \\
K_{1}^{*}
\end{array}\right]<0 .
$$

Making use of Lemma 2 with $\varepsilon=1$, we have

$$
\begin{aligned}
& -C^{T} V_{1}^{* T} V_{1}^{*} C \leq\left(V_{1 c}^{n}\right)^{T} V_{1 c}^{n}-\left(V_{1 c}^{n}\right)^{T} V_{1}^{*} C-C^{T} V_{1}^{* T} V_{1 c}^{n}, \\
& -C^{T} V_{2}^{* T} V_{2}^{*} C \leq\left(V_{2 c}^{n}\right)^{T} V_{2 c}^{n}-\left(V_{2 c}^{n}\right)^{T} V_{2}^{*} C-C^{T} V_{2}^{* T} V_{2 c}^{n}, \\
& -K_{1}^{* T} K_{1}^{*} \leq\left(K_{1}^{n}\right)^{T} K_{1}^{n}-\left(K_{1}^{n}\right)^{T} V_{1}^{*} F_{y} F_{\theta}-F_{\theta}^{T} F_{y}^{T} V_{1}^{* T} K_{1}^{n} .
\end{aligned}
$$

Applying Schur complement to (40) and changing variables $\varphi_{1}\left(V_{1}^{*}, V_{1 c}^{n}\right), \varphi_{2}\left(V_{2}^{*}, V_{2 c}^{n}\right)$, and $\varphi_{3}\left(K_{1}^{*}, K_{1}^{n}\right)$, the LMI (32) is obtained. To overcome the infeasibility of (33), the same variables as those selected in Theorem 3 are used. It completes the proof.

Corollary 5. The system is asymptotically stable and satisfies (16) if there exists symmetric positive matrices $P, Q, V_{1}^{*}, V_{2}^{*}$, $\Phi_{1}^{*}, \Phi_{2}^{*}$, and $\Phi_{3}^{*}$ such that the LMIs (26) and (32) hold.

Remark 6. It is desired to obtain a reference residual system which has maximum sensitivity to the fault as well as the minimum sensitivity to the exogenous signal. This aim can be formulated by performance index defined by inf $\alpha / \beta$. To this end, an iterative optimization method presented in [6] is developed for the proposed structure. The procedures of this method are as follows.

(1) Choose appropriate values of $\alpha$ and $\beta$.

(2) Solve the LMI (26), and find a feasible solution for $P$, $\mathrm{Q}, V_{1}^{*}, V_{2}^{*}, \Phi_{1}^{*}, \Phi_{2}^{*}$, and $\Phi_{3}^{*}$ matrices.

(3) Set $V_{1 c}^{n}=V_{1}^{* n-1} C, V_{2 c}^{n}=V_{2}^{* n-1} C, K_{11}^{n}=V_{1}^{* n-1} F_{y} F_{\theta}$, and $K_{12}^{n}=V_{2}^{* n-1} F_{y} F_{\theta}$. Then, solve (26) and (32) by increasing $n$ to find a feasible solution for $P, \mathrm{Q}, V_{1}^{*}$, $V_{2}^{*}, \Phi_{1}^{*}, \Phi_{2}^{*}$, and $\Phi_{3}^{*}$.

(4) Increase $\beta$ and decrease $\alpha$ and go to step 2. Continue this procedure until the feasible solution cannot be found for LMIs (26) and (32).

(b) Robust UIO Design (Step 2). As mentioned before, the residual signal generator system is obtained by minimizing (17). To this end, Theorem 8 is presented which guarantees that the overall system (19) is asymptotically stable and performance index (17) is minimized. Before presenting Theorem 8, the following theorem is presented which helps prove Theorem 8.

Theorem 7. For a given $\gamma>0$ and the system (37), if there exist symmetric positive matrices $P, Q$ and constants $\varepsilon_{1}, \varepsilon_{2}$, and $\varepsilon_{3}$ such that the LMI (44) holds, then the system (37) is asymptotically stable and $\|v(t)\|_{2} \leq \gamma\|u(t)\|_{2}$ :

$$
\begin{aligned}
& \dot{\chi}(t)=(\widetilde{A}+\Delta \widetilde{A}) \chi(t)+\left(\widetilde{A}_{d}+\Delta \widetilde{A}_{d}\right) \chi(t-\tau)+\left(\widetilde{B}_{u}+\Delta \widetilde{B}_{u}\right) u(t), \\
& v(t)=\widetilde{C}_{1} \chi(t)+\widetilde{C}_{2} \chi(t-\tau)+\widetilde{D} u(t), \\
& {\left[\begin{array}{ccccccc}
P \widetilde{A}+\widetilde{A}^{T} P+Q+\varepsilon_{1}^{-1} \widetilde{N}_{1}^{T} \widetilde{N}_{1} & P \widetilde{A}_{d} & P \widetilde{B}_{u} & \widetilde{C}_{1}^{T} & P \widetilde{M}_{1} & P \widetilde{M}_{2} & P \widetilde{M}_{3} \\
* & -Q+\varepsilon_{2}^{-1} \widetilde{N}_{2}^{T} \widetilde{N}_{2} & \mathbf{0} & \widetilde{C}_{2}^{T} & \mathbf{0} & \mathbf{0} & \mathbf{0} \\
* & * & -\gamma^{2} I+\varepsilon_{3}^{-1} \widetilde{N}_{3}^{T} \widetilde{N}_{3} & \widetilde{D}^{T} & \mathbf{0} & \mathbf{0} & \mathbf{0} \\
* & * & * & -I & \mathbf{0} & \mathbf{0} & \mathbf{0} \\
* & * & * & * & -\varepsilon_{1}^{-1} I & \mathbf{0} & \mathbf{0} \\
* & * & * & * & * & -\varepsilon_{2}^{-1} I & \mathbf{0} \\
* & * & * & * & * & * & -\varepsilon_{3}^{-1} I
\end{array}\right]<\mathbf{0} .}
\end{aligned}
$$


Proof. Define the following Lyapunov-Krasovskii function:

$$
V(t)=\chi^{T}(t) P \chi(t)+\int_{t-\tau}^{t} \chi^{T}(s) Q \chi(s) d s .
$$

The performance index $\|v(t)\|_{2} \leq \gamma\|u(t)\|_{2}$ can be written

$$
\begin{aligned}
J_{v}=\int_{0}^{\infty} & \left(v^{T}(t) v(t)-\gamma^{2} u^{T}(t) u(t)+\dot{V}(t)\right) d t \\
& +V(0)-V(\infty) .
\end{aligned}
$$

Assume $\chi(t)=\mathbf{0}$ for $t \in[-\tau, 0]$. Since $V(\infty)>0$, we have

$$
J_{v} \leq \int_{0}^{\infty}\left(v^{T}(t) v(t)-\gamma^{2} u^{T}(t) u(t)+\dot{V}(t)\right) d t
$$

$$
\begin{aligned}
& J_{r_{e}} \leq \int_{0}^{\infty}\left[\begin{array}{c}
\chi(t) \\
\chi(t-\tau) \\
u(t)
\end{array}\right]^{\left[\begin{array}{ccc}
P(\widetilde{A}+\Delta \widetilde{A})+(\widetilde{A}+\Delta \widetilde{A})^{T} P+Q+\widetilde{C}_{1}^{T} \widetilde{C}_{1} P\left(\widetilde{A}_{d}+\Delta \widetilde{A}_{d}\right)+\widetilde{C}_{1}^{T} \widetilde{C}_{2} & P\left(\widetilde{B}_{u}+\Delta \widetilde{B}_{u}\right)+\widetilde{C}_{1}^{T} \widetilde{D}^{*} \\
* & -Q+\widetilde{C}_{2}^{T} \widetilde{C}_{2} & 0 \\
* & * & -\gamma^{2} I+\widetilde{D}^{T} \widetilde{D}
\end{array}\right]} \\
& \times\left[\begin{array}{c}
\chi(t) \\
\chi(t-\tau) \\
u(t)
\end{array}\right] d t
\end{aligned}
$$

Taking derivative from (45) and considering (37) yield

$\Xi<0$ implies $J_{r_{e}}<0$. The $\Xi<0$ can be written as

$$
\begin{gathered}
{\left[\begin{array}{ccc}
P \widetilde{A}+\widetilde{A}^{T} P+Q & P \widetilde{A}_{d} & P \widetilde{B}_{u} \\
* & -Q & \mathbf{0} \\
* & * & -\gamma^{2} I
\end{array}\right]} \\
+\left[\begin{array}{c}
\widetilde{C}_{1}^{T} \\
\widetilde{C}_{2}^{T} \\
\widetilde{D}^{T}
\end{array}\right] I\left[\begin{array}{lll}
\widetilde{C}_{1} & \widetilde{C}_{2} & \widetilde{D}
\end{array}\right] \\
+\left[\begin{array}{ccc}
P \Delta \widetilde{A}+\Delta \widetilde{A}^{T} P & P \Delta \widetilde{A} & P \Delta \widetilde{B}_{u} \\
* & \mathbf{0} & \mathbf{0} \\
* & & \mathbf{0}
\end{array}\right]<0 .
\end{gathered}
$$

Using Lemma 2, one can write the following inequality:

$$
\begin{aligned}
& {\left[\begin{array}{ccc}
P \Delta \widetilde{A}+\Delta \widetilde{A}^{T} P & P \Delta \widetilde{A}_{d} & P \Delta \widetilde{B}_{u} \\
* & \mathbf{0} & \mathbf{0} \\
* & * & \mathbf{0}
\end{array}\right]} \\
& \leq\left[\begin{array}{ccc}
P \widetilde{M}_{1} & P \widetilde{M}_{2} & P \widetilde{M}_{3} \\
\mathbf{0} & \mathbf{0} & \mathbf{0} \\
\mathbf{0} & \mathbf{0} & \mathbf{0}
\end{array}\right]\left[\begin{array}{ccc}
\varepsilon_{1} I & \mathbf{0} & \mathbf{0} \\
\mathbf{0} & \varepsilon_{2} I & \mathbf{0} \\
\mathbf{0} & \mathbf{0} & \varepsilon_{3} I
\end{array}\right] \\
& \times\left[\begin{array}{ccc}
P \widetilde{M}_{1} & P \widetilde{M}_{2} & P \widetilde{M}_{3} \\
\mathbf{0} & \mathbf{0} & \mathbf{0} \\
\mathbf{0} & \mathbf{0} & \mathbf{0}
\end{array}\right]^{T}+\left[\begin{array}{ccc}
\widetilde{N}_{1}^{T} & \mathbf{0} & \mathbf{0} \\
\mathbf{0} & \widetilde{N}_{2}^{T} & \mathbf{0} \\
\mathbf{0} & \mathbf{0} & \widetilde{N}_{3}^{T}
\end{array}\right] \\
& \times\left[\begin{array}{ccc}
\varepsilon_{1} I & \mathbf{0} & \mathbf{0} \\
\mathbf{0} & \varepsilon_{2} I & \mathbf{0} \\
\mathbf{0} & \mathbf{0} & \varepsilon_{3} I
\end{array}\right]^{-1}\left[\begin{array}{ccc}
\widetilde{N}_{1} & \mathbf{0} & \mathbf{0} \\
\mathbf{0} & \widetilde{N}_{2} & \mathbf{0} \\
\mathbf{0} & \mathbf{0} & \widetilde{N}_{3}
\end{array}\right] .
\end{aligned}
$$

Considering (49), (50) and using Schur complement (48) lead to (44). It completes the proof.

Theorem 8. For a given $\gamma>0$, if there exist symmetric positive matrices $P_{1}, P_{2}, P_{3}, Q_{1}, Q_{2}$, and $Q_{3}$, matrices $\Phi_{1}, \Phi_{2}$, and $\Phi_{3}$, and constants $\varepsilon_{1}, \varepsilon_{2}$, and $\varepsilon_{3}$ such that LMI $\left[s_{i j}\right]_{14 \times 14}<0$ holds, then the overall system (19) is asymptotically stable, and $J_{r_{e}}<\gamma$. The observer matrices are calculated by considering (4), (5), and conditions 2 to 5 , and $Y=P_{1}^{-1} \Phi_{1}, L_{2}=P_{1}^{-1} \Phi_{2}$, and $L_{3}=$ $P_{1}^{-1} \Phi_{3}$. The LMI coefficients are defined as

$$
\begin{aligned}
& s_{1,1}=P_{1} F+\left(P_{1} F\right)^{T}+Q_{1}, \quad s_{1,4}=P_{1} G, \quad s_{1,8}=P_{1} \bar{F}, \\
& s_{1,10}=P_{1} \bar{R}, \quad s_{1,11}=C^{T} V_{1}^{T}, \quad s_{1,12}=P_{1}\left(T M_{1}\right), \\
& s_{1,13}=P_{1}\left(T M_{2}\right), \quad s_{1,14}=P_{1}\left(T M_{3}\right), \\
& s_{2,2}=P_{2} F^{*}+\left(P_{2} F^{*}\right)^{T}+Q_{2}, \quad s_{2,5}=P_{2} G^{*}, \quad s_{2,8}=P_{2} \bar{F}^{*}, \\
& s_{2,10}=P_{2} \bar{R}^{*}, \quad s_{2,11}=-C^{T} V_{1}^{* T}, \\
& s_{3,3}=P_{3} A+\left(P_{3} A\right)^{T}+Q_{3}+\varepsilon_{1}^{-1} N_{1}^{T} N_{1}, \quad s_{3,6}=P_{3} A_{d}, \\
& s_{3,7}=P_{3} B, \quad s_{3,8}=P_{3} K_{3}, \quad s_{3,9}=P_{3} E, \quad s_{3,10}=P_{3} K_{4}, \\
& s_{3,12}=P_{3}\left(M_{1}\right), \quad s_{3,13}=P_{3}\left(M_{2}\right), \quad s_{3,14}=P_{3}\left(M_{3}\right), \\
& s_{4,4}=-Q_{1}, \quad s_{4,11}=C^{T} V_{2}^{T}, \\
& s_{5,5}=-Q_{2}, \quad s_{5,11}=C^{T} V_{2}^{* T}, \quad s_{6,6}=-Q_{3}+\varepsilon_{2}^{-1} N_{2}^{T} N_{2}, \\
& s_{7,7}=-\gamma I+\varepsilon_{3}^{-1} N_{3}^{T} N_{3}, \quad s_{8,8}=-\gamma I, \quad s_{8,11}=K_{1}^{T}-K_{1}^{* T}, \\
& s_{9,9}=-\gamma I, \quad s_{10,10}=-\gamma I, \quad s_{10,11}=K_{2}^{T}-K_{2}^{* T}, \\
& s_{11,11}=-I, \quad s_{12,12}=-\varepsilon_{1}^{-1} I,
\end{aligned}
$$




$$
s_{13,13}=-\varepsilon_{2}^{-1} I, \quad s_{14,14}=-\varepsilon_{3}^{-1} I, \quad \text { where }
$$

otherwise $s_{i, j}=\mathbf{0}$,

$$
\begin{gathered}
P_{1} T=P_{1}\left(I-\Theta_{1} C\right)-\Phi_{1}\left(\Theta_{2} C\right), \\
P_{1} F=P_{1}\left(A-\Theta_{1} C A\right)-\Phi_{1}\left(\Theta_{2} C A\right)-\Phi_{2} C, \\
P_{1} G=P_{1}\left(A_{d}-\Theta_{1} C A_{d}\right)-\Phi_{1}\left(\Theta_{2} C A_{d}\right)-\Phi_{3} C, \\
P_{1} \bar{F}=\left[P_{1}\left(F_{x} F_{\theta}-\Theta_{1} C F_{x} F_{\theta}\right)-\Phi_{1}\left(\Theta_{2} C F_{x} F_{\theta}\right)-\Phi_{2} F_{y} F_{\theta}-P_{1}\left(\Theta_{1} F_{y} F_{\theta} A_{\theta}\right)-\Phi_{1}\left(\Theta_{2} F_{y} F_{\theta} A_{\theta}\right)-\Phi_{3} F_{y} F_{\theta}\right], \\
P_{1} \bar{R}=\left[P_{1}\left(R-\Theta_{1} C R\right)-\Phi_{1}\left(\Theta_{2} C R\right)-\Phi_{2} D-\Phi_{3} D-P_{1}\left(\Theta_{1} D\right)-\Phi_{1}\left(\Theta_{2} D\right)\right] .
\end{gathered}
$$

Proof. In Theorem 7 assume that $P=\operatorname{diag}\left(\begin{array}{lll}P_{1} & P_{2} & P_{3}\end{array}\right)$ and $Q=\operatorname{diag}\left(Q_{1} \quad Q_{2} \quad Q_{3}\right)$. Then, using system dynamic (19) it is straight forward to see that $s_{i j}$ are the same as (37). Without considering (52), the inequality (51) includes nonlinear terms of $P Y, P L_{2}$, and $P L_{3}$ which lead the LMI to be infeasible. To overcome this problem, define $\Phi_{1}=P_{1} Y, \Phi_{2}=P_{1} L_{2}$, and $\Phi_{3}=P_{1} L_{3}$. Using conditions 2,3 and (23) it can be seen that (52) makes the obtained LMI feasible. It completes the proof.

Remark 9. It should be noted that the present work differs from [18] in the following perspectives.

(a) The results in [18] are obtained without considering dynamic characteristic for fault; however, the current results are achieved by considering dynamic characteristic that is modeled by (3). Hence, the design procedure in [18] is not applicable for the current case.

(b) The residual signal $r(t)$ is constructed based on (14) which uses both estimation error and delay in estimation error; however, the residual signal in [18] is constructed using estimation error. Since two design parameters $V_{1}$ and $V_{2}$ appear in the LMIs, the obtained LMIs are more flexible.

Remark 10. After designing the FDI system, residual evaluation methods and appropriate level of threshold should be selected to take a decision about the occurrence of fault. According to (13) and (14), the residual signal for fault-free system $r^{0}(t)$ satisfies the following equation:

$$
\begin{aligned}
\left\|r^{0}(t)\right\|_{2} & =\left\|r_{\bar{n}}(t)+r_{u}(t)\right\|_{2} \\
& \leq\left\|r_{\bar{n}}(t)\right\|_{2}+\left\|r_{u}(t)\right\|_{2} \leq J_{\mathrm{th}, \bar{n}}+J_{\mathrm{th}, u},
\end{aligned}
$$

where

$$
\begin{aligned}
& J_{\text {th }, \bar{n}}=\sup _{\left(\Delta A, \Delta B, \Delta A_{d}\right) \in \Omega_{i}}\left\|r_{\bar{n}}(t)\right\|_{2}, \\
& J_{\text {th }, u}=\sup _{\left(\Delta A, \Delta B, \Delta A_{d}\right) \in \Omega_{i}}\left\|r_{u}(t)\right\|_{2} .
\end{aligned}
$$

$J_{\text {th, }, \bar{n}}$ can be computed offline, and under the assumption that $\bar{n} \in L_{2}$ we have $\sup _{\left(\triangle A, \Delta B, \Delta A_{d}\right) \in \Omega_{i}}\left\|r_{\bar{n}}(t)\right\|_{2}=M_{\bar{n}}$. Since the signal $u$ is supposed to be known online, the value of $J_{\text {th, }, u}$ can be determined online by $J_{\text {th }, u}=\gamma_{u}\|u(t)\|_{2}$, where $\gamma_{u}=$ $\sup _{\left(\Delta A, \Delta B, \Delta A_{d}\right) \in \Omega_{i}}\left(\left\|r_{u}(t)\right\|_{2}\right) /\left(\|u(t)\|_{2}\right) \cdot \gamma_{u}$ can be computed by Theorem 7 . Therefore, the threshold value can be evaluated by

$$
J_{\text {th }}=M_{\bar{n}}+\gamma_{u}\|u(t)\|_{2}
$$

Since values of (53) and (54) increase by passing the time and, consequently, need more memory in real application, one can use root-mean-square (RMS) norm of $r(t)$, defined in (56), to detect the fault signals:

$$
\|r(t)\|_{2}^{T}=\int_{t_{1}}^{t_{2}} r^{T}(t) r(t) d t, \quad T=t_{2}-t_{1},
$$

where $T$ is designed parameter.

\section{Simulation Results}

The main objective of this section is to investigate the effectiveness of the designed UIO. To this end, a numerical example is used and simulation results are presented. Consider a system which is defined by (1) with the following matrices:

$$
\begin{array}{cc}
A=\left[\begin{array}{ccc}
-3.8 & 1.5 & -0.5 \\
0.5 & -3 & 1 \\
-0.3 & 0.7 & -2.4
\end{array}\right], \quad A_{d}=\left[\begin{array}{ccc}
0.4 & 0.1 & -0.2 \\
0.1 & -0.8 & 0.2 \\
0.7 & -0.1 & 0.5
\end{array}\right], \\
B=\left[\begin{array}{c}
0.1 \\
0.2 \\
-0.4
\end{array}\right], \quad F_{x}=\left[\begin{array}{c}
0.6 \\
-0.5 \\
0.4
\end{array}\right], \quad E=\left[\begin{array}{c}
-0.4 \\
0.1 \\
-0.3
\end{array}\right], \\
C=\left[\begin{array}{lll}
1 & 0 & 0 \\
0 & 1 & 0 \\
0 & 0 & 1
\end{array}\right], \quad F_{y}=\left[\begin{array}{c}
0.2 \\
0.8 \\
-1.2
\end{array}\right], \quad R=\left[\begin{array}{c}
0.1 \\
0.2 \\
-0.4
\end{array}\right], \\
D=\left[\begin{array}{c}
0.9 \\
0.2 \\
0.7
\end{array}\right] .
\end{array}
$$


Uncertainties are also defined by the following matrices in (2):

$$
\begin{gathered}
M_{1}=\left[\begin{array}{l}
0.1 \\
0.2 \\
0.1
\end{array}\right], \quad M_{2}=\left[\begin{array}{c}
0.1 \\
0 \\
-0.1
\end{array}\right], \quad M_{3}=\left[\begin{array}{c}
-0.1 \\
0.2 \\
0.1
\end{array}\right], \\
N_{1}=\left[\begin{array}{lll}
0 & 0.1 & 0.3
\end{array}\right], \\
N_{2}=\left[\begin{array}{lll}
0.1 & 0 & 0
\end{array}\right], \\
N_{3}=0.1 .
\end{gathered}
$$

The dynamic characteristic of fault is considered as

$$
A_{\theta}=0, \quad F_{\theta}=1 \text {. }
$$

The first step to design the fault detection system is to solve the LMIs (26) and (32) in Theorems 3 and 4. The Yalmip LMI toolbox is used to solve the LMIs. To start the iterative optimization method presented in Remark 6, the initial values $\alpha_{\text {int }}=3$ and $\beta_{\text {int }}=0$ are selected. Using this procedure, the following results are obtained:

$$
\begin{aligned}
& V_{1}^{*}=\left[\begin{array}{ccc}
-0.0994 & -0.0532 & 0.2714 \\
-0.0532 & -5.4751 & 1.3859 \\
0.2714 & 1.3859 & -0.7651
\end{array}\right] \text {, } \\
& V_{2}^{*}=\left[\begin{array}{ccc}
-0.6315 & -0.8304 & 0.1871 \\
-0.8304 & 0.6711 & 0.5971 \\
0.1871 & 0.5971 & -0.5845
\end{array}\right] \text {, } \\
& \Phi_{1}^{*}=\left[\begin{array}{ccc}
662.5 & -250.8 & -2628.5 \\
-250.8 & 3855.7 & -281.2 \\
-2628.5 & -281.2 & 2055.8
\end{array}\right] \text {, } \\
& \Phi_{2}^{*}=\left[\begin{array}{ccc}
984.8 & -1453.4 & -842.7 \\
-1453.4 & 2292 & 1253.8 \\
-842.7 & 1253.8 & 733.6
\end{array}\right] \text {, } \\
& \Phi_{3}^{*}=\left[\begin{array}{ccc}
-4.7395 & 13.0831 & 0.7977 \\
13.0831 & -8.5691 & -14.9116 \\
0.7977 & -14.9116 & -3.0645
\end{array}\right] \text {, } \\
& \alpha=2.4, \quad \beta=1 .
\end{aligned}
$$

Using these values, the LMI (51) is solved and the observer dynamic matrices are obtained as follows:

$$
\begin{aligned}
& F=\left[\begin{array}{lll}
-3.6557 & -3.3806 & 2.9608 \\
-0.3878 & -1.4059 & 0.3008 \\
-2.4404 & -3.2245 & 1.9455
\end{array}\right], \\
& G=\left[\begin{array}{lll}
-0.0559 & -0.7284 & -0.1686 \\
-0.0153 & -0.1629 & -0.0350 \\
-0.0445 & -0.557 & -0.1273
\end{array}\right], \\
& H=\left[\begin{array}{l}
0.6878 \\
0.1535 \\
0.5328
\end{array}\right],
\end{aligned}
$$

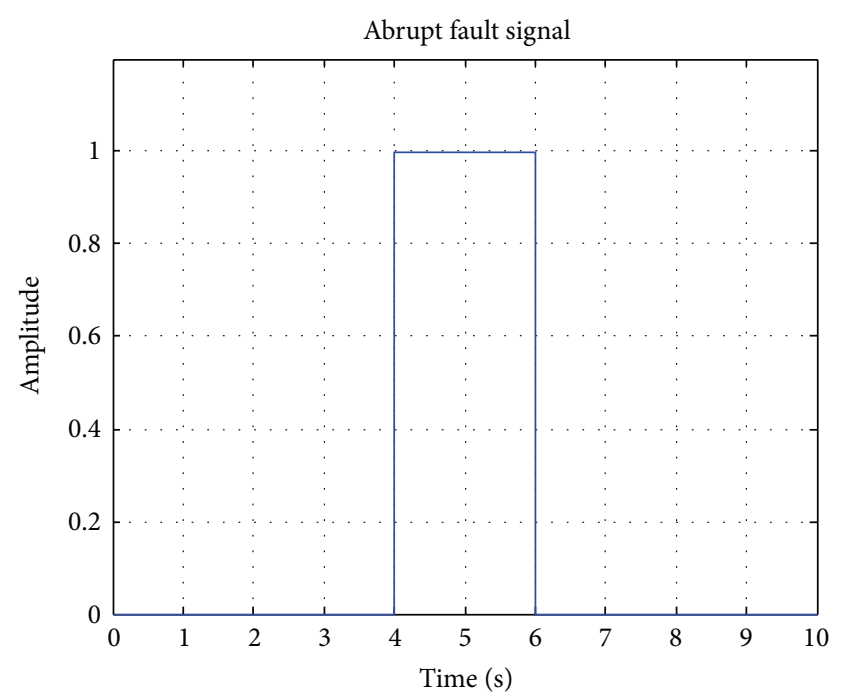

Figure 1: An abrupt fault occurs at $t=5$.

$$
\begin{aligned}
& L_{1}=\left[\begin{array}{ccc}
0.0372 & -1.1695 & 0.8939 \\
-0.2145 & 0.7355 & 0.1978 \\
-0.7462 & -0.9036 & 1.6937
\end{array}\right], \\
& K_{1 z}=\left[\begin{array}{lll}
-0.7701 & -0.1957 & 0.9507 \\
-0.1730 & -0.0567 & 0.2159 \\
-0.5857 & -0.1278 & 0.7254
\end{array}\right], \\
& K_{2 z}=\left[\begin{array}{lll}
0.2122 & -0.3395 & -0.7167 \\
0.0496 & -0.0777 & -0.1591 \\
0.1603 & -0.2648 & -0.5534
\end{array}\right] .
\end{aligned}
$$

To verify the sensitivity of designed UIO, an abrupt fault, shown in Figure 1, occurs in the 4 seconds elapsed from running of the system. The step disturbance signal exerted to the system between 3 to 7 seconds. The noise signal is assumed to be white Gaussian noise with power 0.0005 , and the uncertainty $\Sigma_{i}(t)$ is considered sinusoidal signal. The residual signals are shown in Figure 2 . It can be seen that the residual signals change when the fault occurs; however, the residual signals do not show any sensitivity to the exerted disturbance. The value of threshold $J_{\text {th }}$ is presented in Figure 3. This figure indicates that the fault is detected rapidly and the difference between threshold and norm of faulty residual signal is high enough to detect the occurrence of fault in the system.

The RMS of residual signals (56) has been depicted in Figure 4. It can be seen that the RMS of faulty signals suddenly changes in contrast to RMS of fault-free signals. Therefore, the occurrence of fault can be effectively realized.

\section{Conclusions}

In this paper, a novel UIO-based residual generator is developed for robust fault detection purposes. The developed method is applicable to a variety of linear uncertain timedelay systems. The proposed approach is able to decouple 


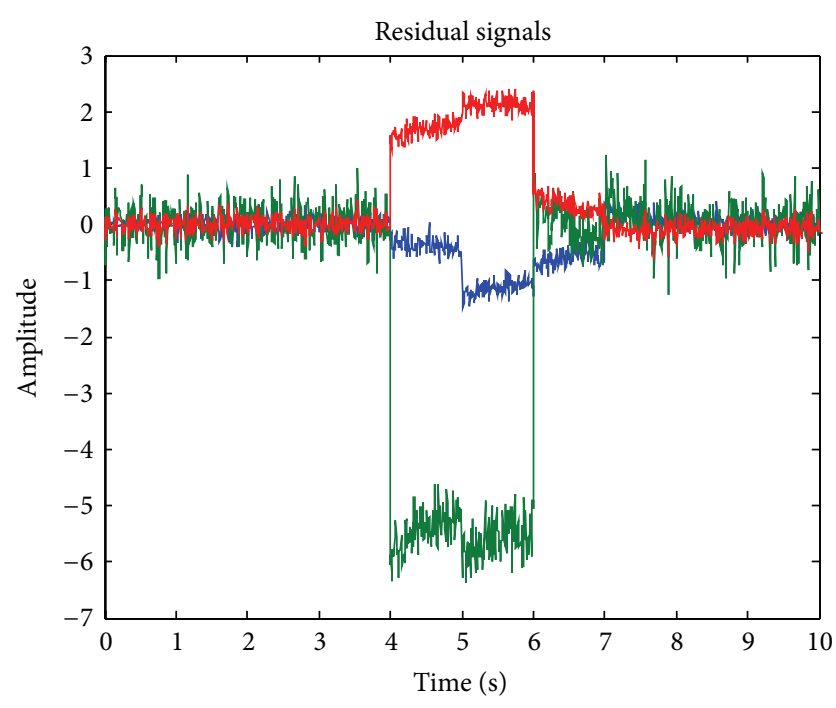

FIgURE 2: Residual signals of UIO fault detection filter.

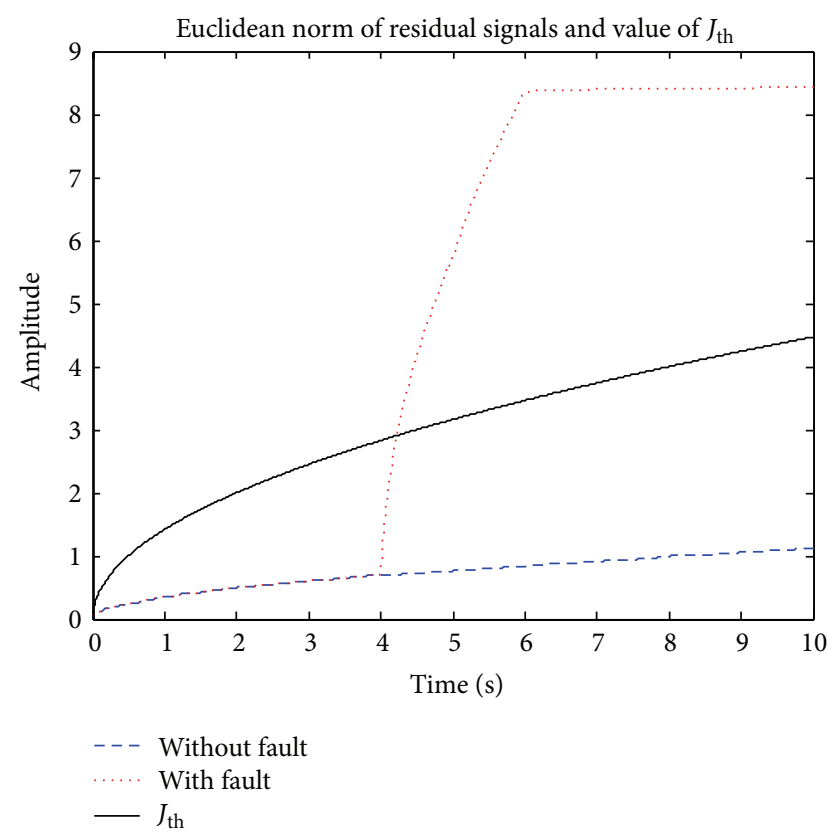

FIGURE 3: Euclidean norm of residual fault-free and faulty signals and $J_{\text {th }}$.

thoroughly exogenous disturbances while minimizing uncertainties and noise effects. The fault effect is also maximized at the same time. To this end, first, the optimal fault detection filter is designed for system without considering uncertainties. Then, the fault detection filter is designed so that the $H_{\infty}$ norm between the fault detection filter and the optimal one is minimized. Superiority of the proposed approach has been verified through a numerical example. Simulation results show that the proposed approach is able to detect dynamic faults. As a future work, one can extend this approach to nonlinear systems and descriptor system. Moreover, developing

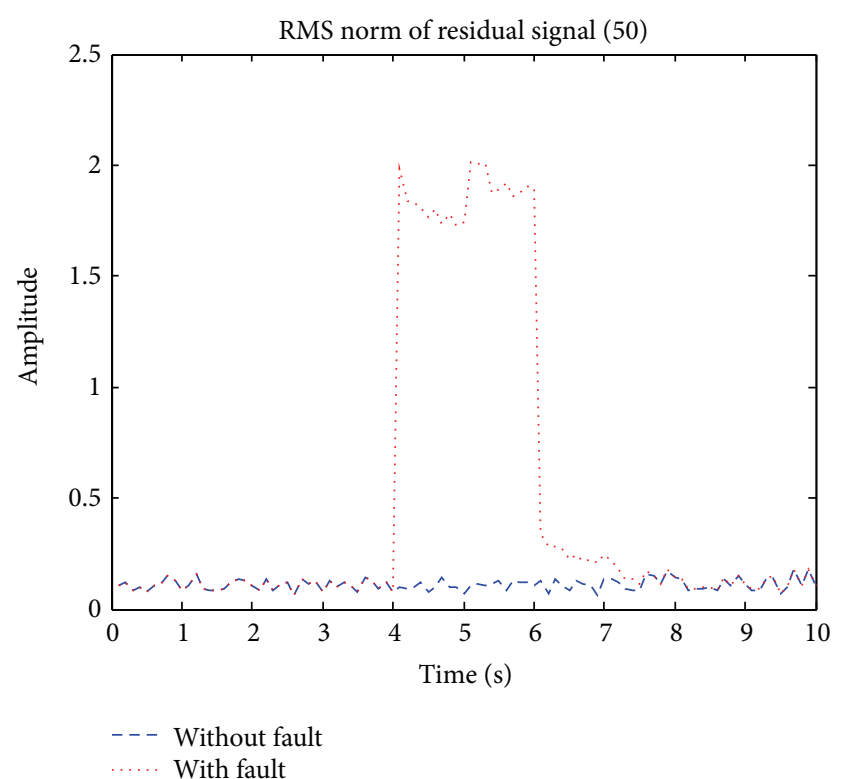

FIGURE 4: Detecting occurrence of fault using RMS norm of $\|r(t)\|_{2}^{T}$.

the data framework for designing unknown input observer for time-delay system is an interesting area.

\section{Conflict of Interests}

The authors declare that there is no conflict of interests regarding the publication of this paper.

\section{References}

[1] L. Zhang, E.-K. Boukas, L. Baron, and H. R. Karimi, "Fault detection for discrete-time Markov jump linear systems with partially known transition probabilities," International Journal of Control, vol. 83, no. 8, pp. 1564-1572, 2010.

[2] D. Dongsheng, B. Jiang, P. Shi, and H. R. Karimi, "Fault detection for continues-time switched systems under asynchronous switching," International Journal of Robust and Nonlinear Control, 2013.

[3] J. Yu, G. Sun, and H. R. Karimi, "Fault reconstruction based cascaded sliding mode observers for descriptor linear systems," Mathematical Problems in Engineering, vol. 2012, Article ID 623426, 20 pages, 2012.

[4] S. Yin, X. Yang, and H. R. Karimi, "Data-driven adaptive observer for fault diagnosis," Mathematical Problems in Engineering, vol. 2012, Article ID 832836, 21 pages, 2012.

[5] J. Chen and R. J. Patton, Robust Model-Based Fault Diagnosis for Dynamic Systems, Kluwer Academic Publishers, 1999.

[6] J. Guo, X. Huang, and Y. Cui, "Design and analysis of robust fault detection filter using LMI tools," Computers and Mathematics with Applications, vol. 57, no. 11-12, pp. 1743-1747, 2009.

[7] M. Zhong, S. X. Ding, T. Bingyong, T. Jeinsch, and M. Sader, "An LMI approach to design robust fault detection observers," in Proceedings of the 4th World Congress on Intelligent Control and Automation, pp. 2705-2709, June 2002. 
[8] M. Zhong, S. X. Ding, J. Lam, and H. Wang, "An LMI approach to design robust fault detection filter for uncertain LTI systems," Automatica, vol. 39, no. 3, pp. 543-550, 2003.

[9] T. Li, L. Wu, and X. Wei, "Robust fault detection filter design for uncertain LTI systems based on new bounded real lemma," International Journal of Control, Automation and Systems, vol. 7, no. 4, pp. 644-650, 2009.

[10] M. Zhong, S. X. Ding, J. Lam, and C. Zhang, "Fault detection filter design for LTI system with time delays," in Proceedings of the 42nd IEEE Conference on Decision and Control, pp. 14671472, December 2003.

[11] L. Bai, Z. Tian, and S. Shi, "Design of $\mathrm{H}_{\infty}$ robust fault detection filter for linear uncertain time-delay systems," ISA Transactions, vol. 45, no. 4, pp. 491-502, 2006.

[12] M. Zhong, H. Ye, T. Sun, and G. Wang, "An iterative LMI approach to RFDF for linear system with time-varying delays," Asian Journal of Control, vol. 8, no. 1, pp. 86-90, 2006.

[13] H. Wang, C. Wang, and H. Gao, "An LMI approach to robust fault detection filter design for uncertain state-delayed systems," in Advances in Machine Learning and Cybernetics, vol. 3930 of Lecture Notes in Computer Science, pp. 112-121, 2006.

[14] H. R. Karimi, M. Zapateiro, and N. Luo, "A linear matrix inequality approach to robust fault detection filter design of linear systems with mixed time-varying delays and nonlinear perturbations," Journal of the Franklin Institute, vol. 347, no. 6, pp. 957-973, 2010.

[15] M. Witczak, A. Obuchowicz, and J. Korbicz, "Genetic programming based approaches to identification and fault diagnosis of non-linear dynamic systems," International Journal of Control, vol. 75, no. 13, pp. 1012-1031, 2002.

[16] G.-R. Duan and R. J. Patton, "Robust fault detection using Luenberger-type unknown input observers-a parametric approach," International Journal of Systems Science, vol. 32, no. 4, pp. 533-540, 2001.

[17] P. M. Frank and X. Ding, "Survey of robust residual generation and evaluation methods in observer-based fault detection systems," Journal of Process Control, vol. 7, no. 6, pp. 403-424, 1997.

[18] S. Ahmadizadeh, J. Zarei, and H. R. Karimi, "Robust unknown input observer design for linear uncertain time delay systems with application to fault detection," Asian Journal of Control, vol. 16, pp. 1-14, 2014.

[19] W. Chen and M. Saif, "Fault detection and isolation based on novel unknown input observer design," in Proceedings of the American Control Conference, pp. 5129-5134, June 2006.

[20] S. Mondal, G. Chakraborty, and K. Bhattacharyya, "LMI approach to robust unknown input observer design for continuous systems with noise and uncertainties," International Journal of Control, Automation and Systems, vol. 8, no. 2, pp. 210-219, 2010.

[21] G.-Y. Tang and J. Li, "Optimal fault diagnosis for systems with delayed measurements," IET Control Theory and Applications, vol. 2, no. 11, pp. 990-998, 2008.

[22] Y.-M. Fu, G.-R. Duan, and S.-M. Song, "Design of unknown input observer for linear time-delay systems," International Journal of Control, Automation and Systems, vol. 2, no. 4, pp. 530-535, 2004. 


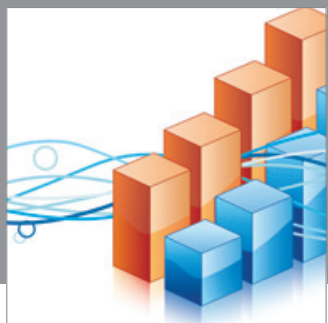

Advances in

Operations Research

mansans

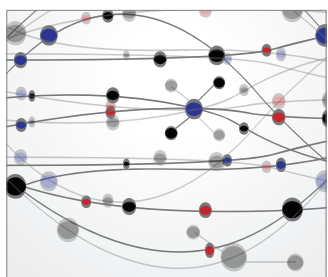

The Scientific World Journal
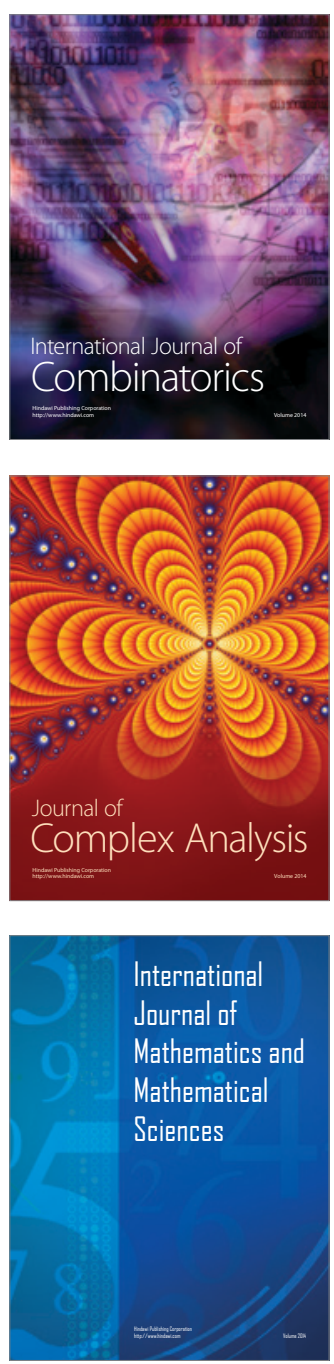
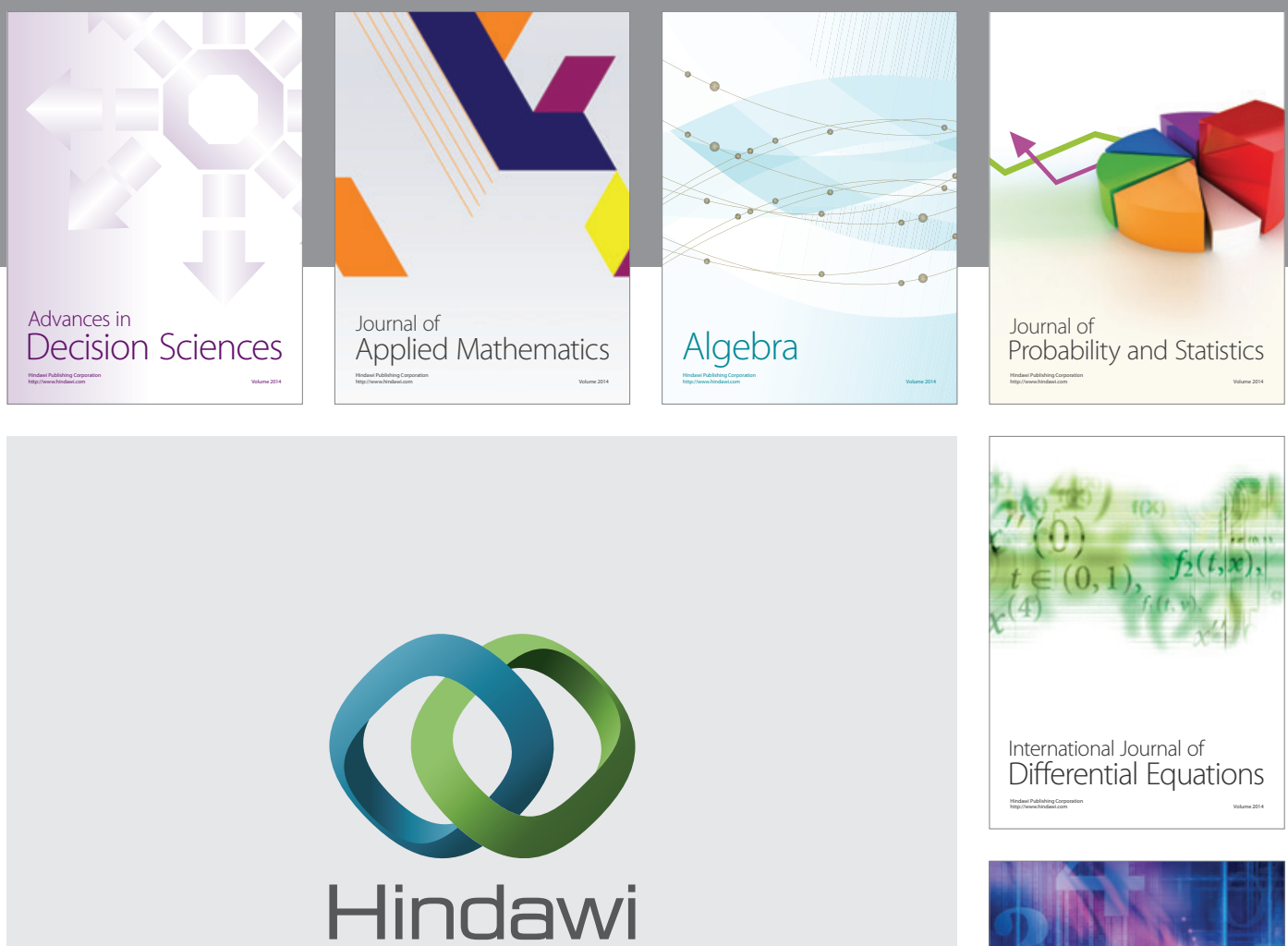

Submit your manuscripts at http://www.hindawi.com
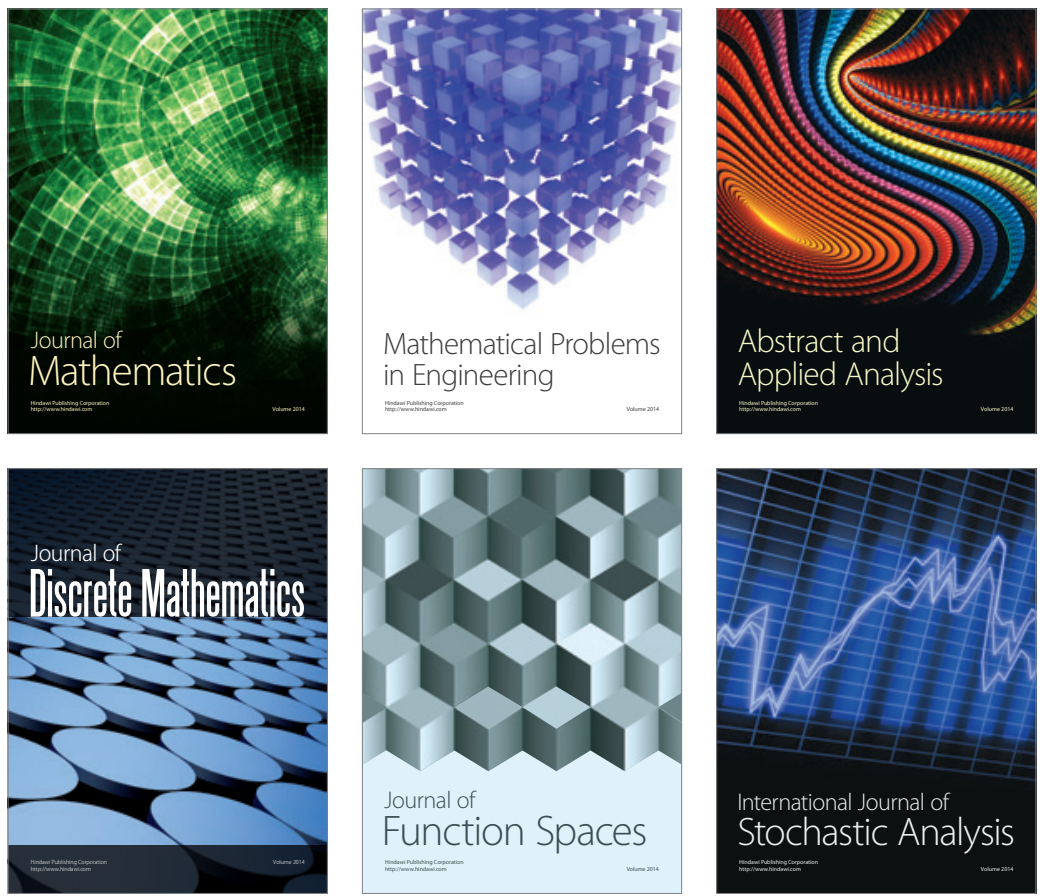

Journal of

Function Spaces




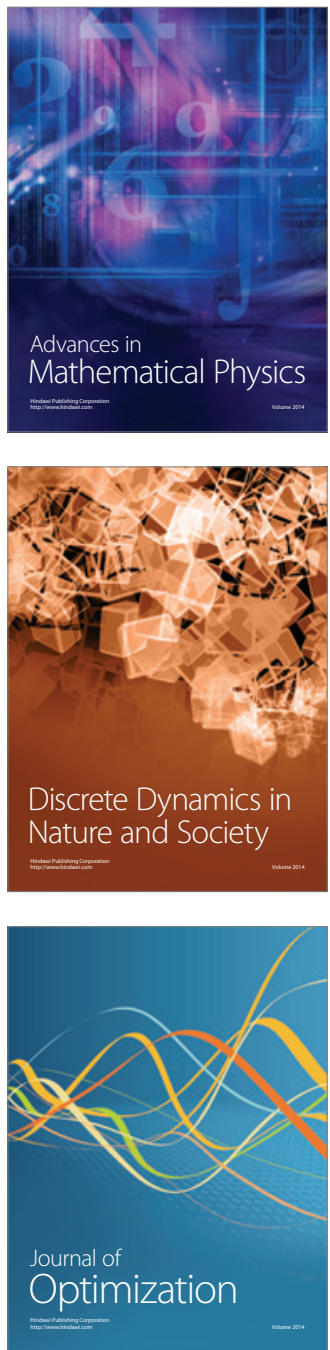